\title{
Pharmacodynamic evaluation of Dezocine injection from Yangtze River Pharmaceutical Group
}

\author{
Rong-Rong Ye ${ }^{1,2}$, Yu-Jun Wang ${ }^{2,3}, \mathrm{Xu} \mathrm{Xu}{ }^{1}$, Yan $\mathrm{Lu}^{1 *}$, Jing-Gen $\mathrm{Liu}^{2,3^{*}}$ \\ ${ }^{1}$ School of Chemical and Environmental Engineering, Shanghai Institute of Technology, Shanghai 201418, China \\ ${ }^{2}$ CAS Key Laboratory of Receptor Research, Shanghai Institute of Materia Medica, Chinese Academy of Sciences and Collaborative \\ Innovation Center for Brain Science, Shanghai 201203, China \\ ${ }^{3}$ University of Chinese Academy of Sciences, Beijing 100049, China
}

\begin{abstract}
Intramuscular injection and intravenous injection are important routes of drug administration, which not only have a fast absorption rate, but also avoid the first pass effect of the drug. In 2009, Yangtze Pharmaceutical Group redeveloped Dezocine(13-Amino-5,6,7,8,9,10,11,12- octahydro-5-methyl-5,11methanobenzocyclodecen-3-ol), molecular formula: $\mathrm{C}_{16} \mathrm{H}_{23} \mathrm{NO}$, in the form of injection. Quickly, Dezocine injection becomes the first choice for perioperative pain management in China and accounts for $45 \%$ of the analgesic market. This study mainly used animal pain models to study the analgesic effects of Dezocine injection. The results indicated that Dezocine produced potent analgesic effect in the hot plate and writhing tests.
\end{abstract}

\section{Introduction}

Pain is one of the reactions that occur when the body is stimulated[1]. Physiological pain is a protective mechanism of body, but pathological pain is the main target of pain treatment intervention[2] such as neuralgia, surgical pain. At present, the mainstream painkillers on the market are opioid analgesics. Morphine is the most commonly used opioids for postoperative pain. However, morphine produced severe side effects, including tolerance[3], addiction[4], respiratory depression[5] and gastrointestinal reaction[6], which limits its clinical use. Initially, Dezocine was recognized as an opioid receptor agonist, with potent analgesic potency but with less side effects[7,8]. After Yangtze River Pharmaceutical Group redeveloped Dezocine in China as an injection, Dezocine quickly occupied analgesic market.

As an analog of pentazocine, as shown in figure 1, Dezocine was considered to be a mixed agonist/antagonist of opioid receptors in the early stage[9], The basis for supporting this conclusion is that naloxone can antagonize in a dose-dependent manner of the Dezocine analgesic in paw pressure experiments[10]. In vitro, competitive binding experiments showed that Dezocine had different affinities with three subtypes of opioid receptors, Kappa $>\mathrm{Mu}>$ Delt[11], many of which have been verified in Gharagozlou's lab[12-14]. However, the functional experiments were somewhat various, despite reports that Dezocine were the Kappa opioid receptor antagonist and $\mathrm{Mu}$ opioid receptor agonist, our lab found that Dezocine was partial Kappa opioid receptor agonist and partial $\mathrm{Mu}$ opioid receptor agonist, both of which have been demonstrated in abdominal constriction test[15].

In recent years, Dezocine shows its ability to inhibiting norepinephrine(NA) and 5-hydroxytryptamine (5-HT) reuptake[11]. Wang's lab has shown that Dezocine mediates mechanical and thermal analgesia in neuralgia models and cancer pain through activation of the $\mathrm{Mu}$ opioid receptor in the spinal and inhibition of NA uptake[16]. Several laboratories have documented the analgesic effects of Dezocine in hot plate pain models, inflammatory pain models[9], tail flick experiments[9], paw pressure experiments[17], and neuropathic pain experiment[16], however, the underlying mechanism was unclear yet. Our laboratory has proved that Dezocine has a significant analgesic effect in the abdominal constriction mouse model. The analgesic $\mathrm{ED}_{50}$ is $0.2 \mathrm{mg} / \mathrm{kg}$. And further proved that in the abdominal constriction test, the analgesic effect of Dezocine is through stimulating Kappa opioid receptor and $\mathrm{Mu}$ opioid receptor. Therefore, in this study, we have two goals:1. Further explore whether the analgesic effect of Dezocine in the abdominal constriction test is related to epinephrine and serotonin receptors; 2. Identify the analgesic mechanism of Dezocine in formalin-induced inflammatory pain.

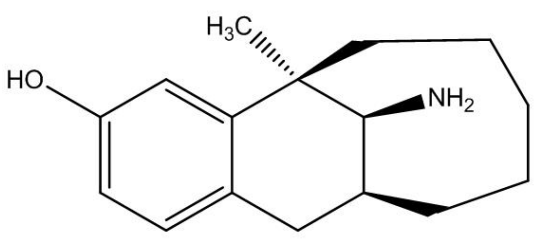

Figure 1. The structure of Dezocine. 


\section{Methods}

\subsection{Materials}

Dezocine: Yangtzi Pharmaceutical Group; $\alpha_{1}$-norepinephrine receptor antagonist: Prazosin (Selleckchem) ; $\alpha_{2}$-norepinephrine receptor antagonist: Yohimbine (Selleckchem); 5-HT $\mathrm{HA}_{1 \mathrm{~A}}$ receptor antagonist: NAD 299 (TOCRIS); 5- HT $_{2 \mathrm{~A}}$ receptor antagonist:Altanserin (TOCRIS); $5-\mathrm{HT}_{2 \mathrm{C}}$ receptor antagonist: RS102221 (TOCRIS); 5- $\mathrm{HT}_{3}$ receptor antagonist:Ondansteron (TOCRIS).

\subsection{Animal}

Kunming strain of mice male and female, 18-22g, obtained from Shanghai Lingchang Biotechnology company. All experiments on laboratory animals are conducted strictly in accordance with the guidelines of the Committee for Animal Protection and use of the Shanghai Institute of Medicine, Chinese Academy of Sciences.

\subsection{Experiment}

2.3.1 Abdominal constriction test. $0.6 \%$ acetic acid solution was injected into the abdominal cavity of mice. The mice showed abdominal contraction, limb tremor, hind legs straight, the body was s-shaped. The number of such symptoms in mice was used as a pain indication to evaluate the analgesic effect of the compound. antinociception $\%=100 *$ (Number of mean control abdominal constriction - Number of test abdominal constriction)/Number of mean control abdominal constriction.

2.3.2 Formalin test. When mice hind paw was injected with 201 of $1.0 \%$ formalin, they showed responses such as foot lifting and licking, which were recorded as indicators of pain. After 60 minutes of observation, antinociception $\%=100 *$ (time of control group - time of administration group)/time of control group.

\section{Statistical Analysis}

All the experimental results were analyzed by Graphpad Prism 6 , the data were all expressed by Mean \pm SEM, the experimental data were analyzed by $\mathrm{T}$-tests, and the difference was statistically significant with $\mathrm{P}<0.05$.

\section{Result}

\subsection{Norepinephrine Receptor Antagonist can attenuated Dezocine analgesia in the abdominal constriction test}

Previous research in our laboratory has shown that Dezocine has a dose dependent analgesic effect in a mouse model of abdominal constriction test through activation of the Kappa opioid receptor and $\mathrm{Mu}$ opioid receptor, with $\mathrm{ED}_{50}$ is $0.2 \mathrm{mg} / \mathrm{kg}$. Therefore, in this trial we selected the dose of $0.25 \mathrm{mg} / \mathrm{kg}$ to conduct experiment to identify Dezocine-induced analgesic effects. Different doses of selective $\alpha_{1}$ - norepinephrine receptor antagonist Prazosin, selective $\alpha_{2}$ - norepinephrine receptor inhibitor Yohimbine, 5- $\mathrm{HT}_{1 \mathrm{~A}}$ receptor antagonist NAD 299, $5-\mathrm{HT}_{2 \mathrm{~A}}$ receptor antagonist Altanserin, 5- $\mathrm{HT}_{2 \mathrm{C}}$ receptor antagonist RS102221 and $5-\mathrm{HT}_{3}$ receptor antagonist Ondansteron were applied in the study. As shown in figure $2,1.25 \mathrm{mg} / \mathrm{kg}$ yohimbine significantly inhibited the analgesic effect of Dezocine. We did not observe the other antagonists can affect Dezocine-indcued antinociceptive action. The results suggest that Dezocine exerts analgesic effects by inhibiting the reuptake of norepinephrine, in addiction to activating opioid receptor.

\subsection{Dezocine produces profound analgesic effects in Formalin induce inflammatory pain models}

To evaluate the analgesic effects of Dezocine in inflammatory pain, formalin-induced pain model was applied. As shown in Table1 and Fig 2a, Dezocine showed a dose-dependent analgesic effect, and the antinociception reached the peak in 15 minutes. 
(a)

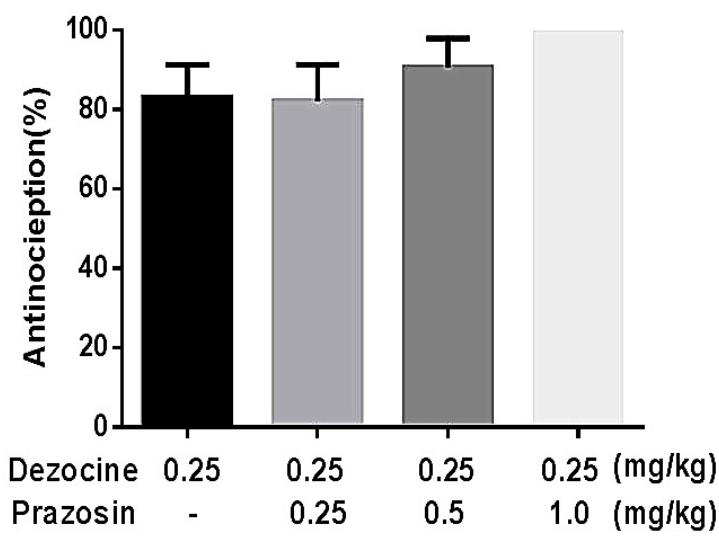

(c)

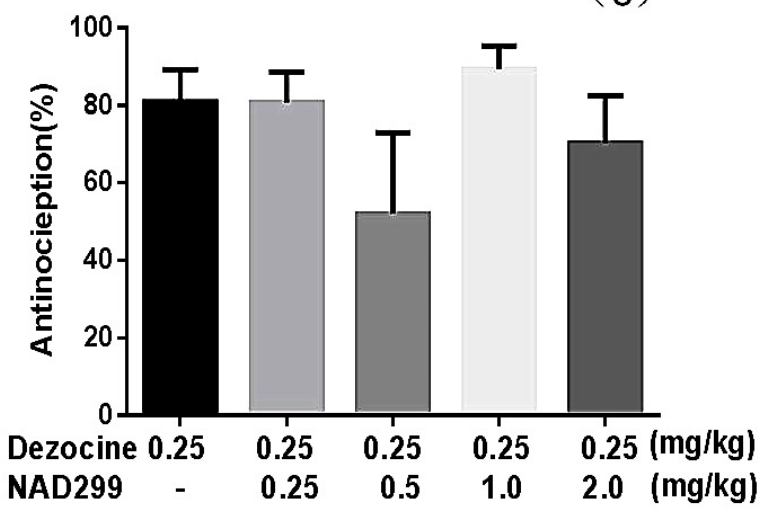

(e)

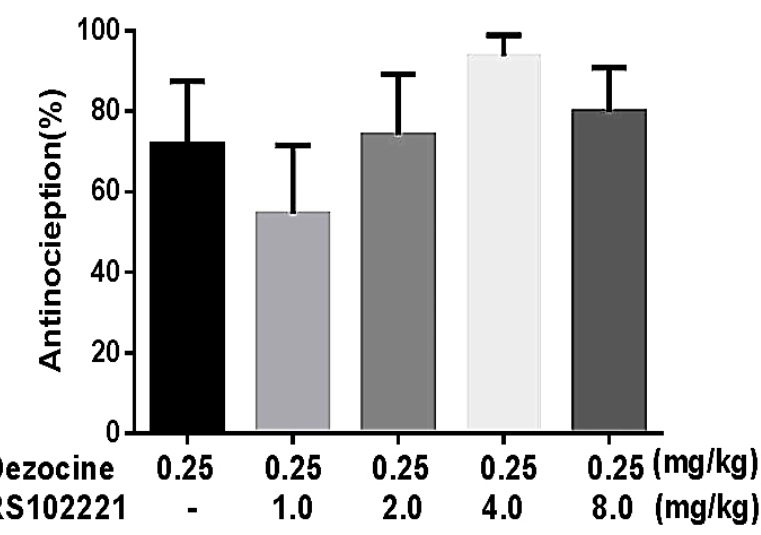

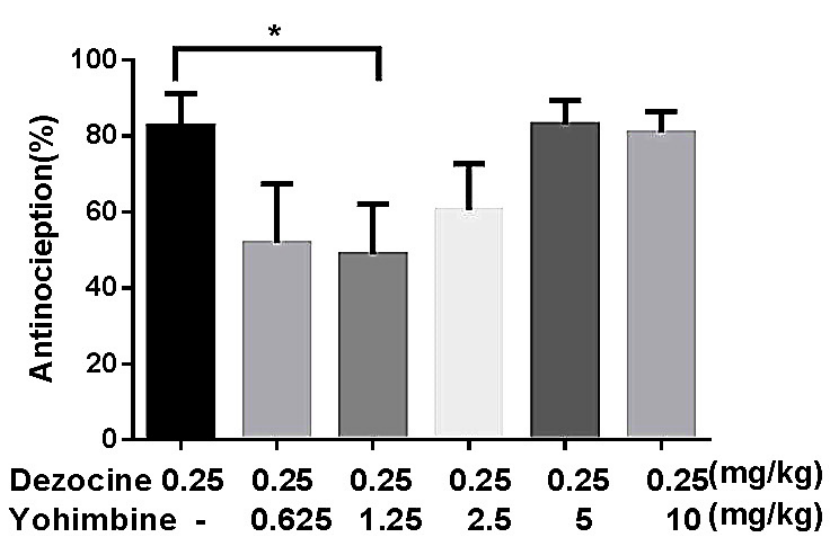

(d)

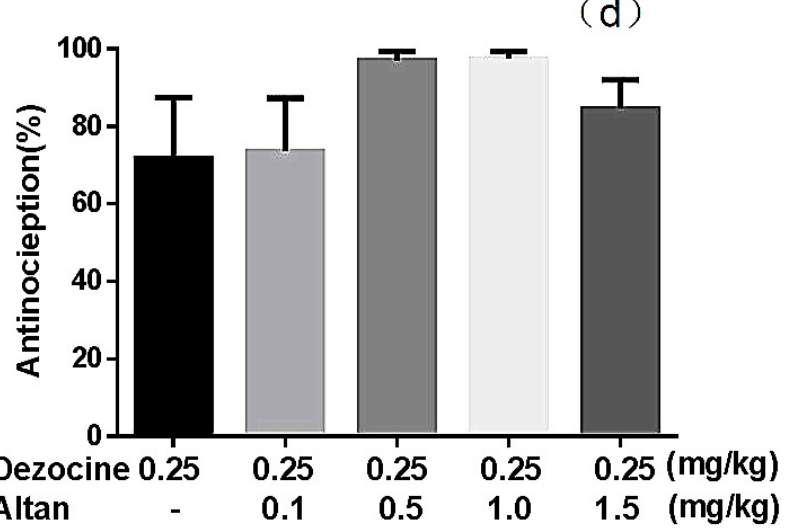

(f)

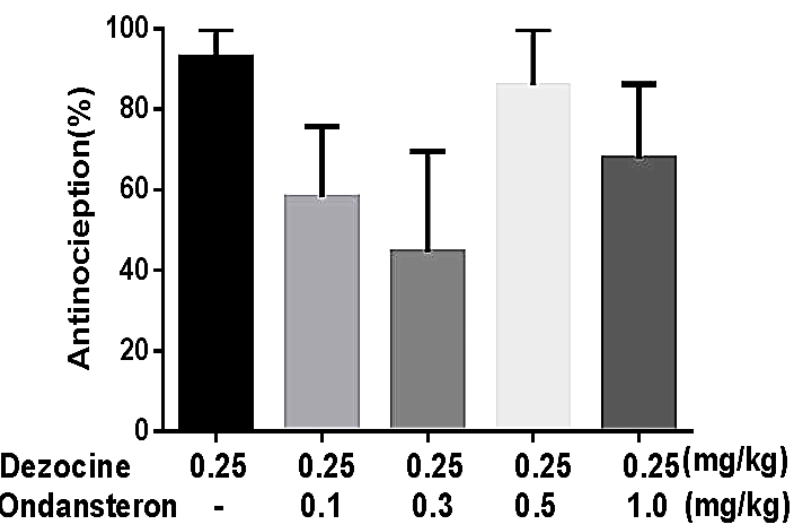

Figure 2: The effects of different norepinephrine receptor antagonists and 5-HT receptor antiagonists on Dezocine-induced analgesic effectgs.

Table 1 Different doses of Dezocine in mice compared with control group of analgesic rate (Mean \pm SEM) in inflammatory pain.

\begin{tabular}{|c|c|c|c|}
\hline Group & $\begin{array}{c}\text { After administration } \\
15 \mathrm{~min} \\
\end{array}$ & $\begin{array}{c}\text { After administration } \\
\text { 30min }\end{array}$ & $\begin{array}{l}\text { After administration } \\
\text { 60min } \\
\end{array}$ \\
\hline Saline $1 \mathrm{ml} / \mathrm{kg}$ & $1.449 \pm 1.449$ & $4.938 \pm 4.938$ & $4.938 \pm 4.938$ \\
\hline Dezocine $0.5 \mathrm{mg} / \mathrm{kg}$ & $18.5 \pm 7.104^{*}$ & $20.04 \pm 7.376$ & $14.78 \pm 5.09$ \\
\hline Dezocine $5 \mathrm{mg} / \mathrm{kg}$ & $37.76 \pm 11.86^{*}$ & $38.31 \pm 11.77^{*}$ & $18.7 \pm 10.05$ \\
\hline Dezocine $20 \mathrm{mg} / \mathrm{kg}$ & $73.45 \pm 11.59 * * * *$ & $74.45 \pm 13^{* * *}$ & $65.31 \pm 12.99 * * *$ \\
\hline
\end{tabular}


(a)

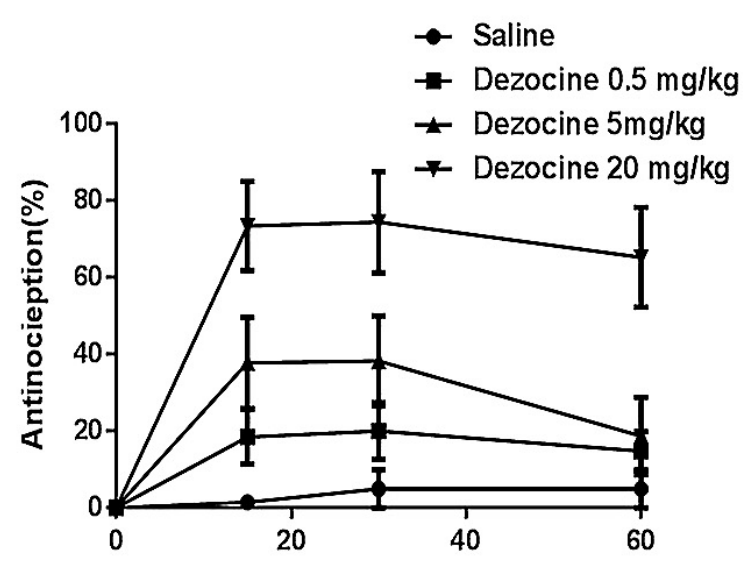

(c)

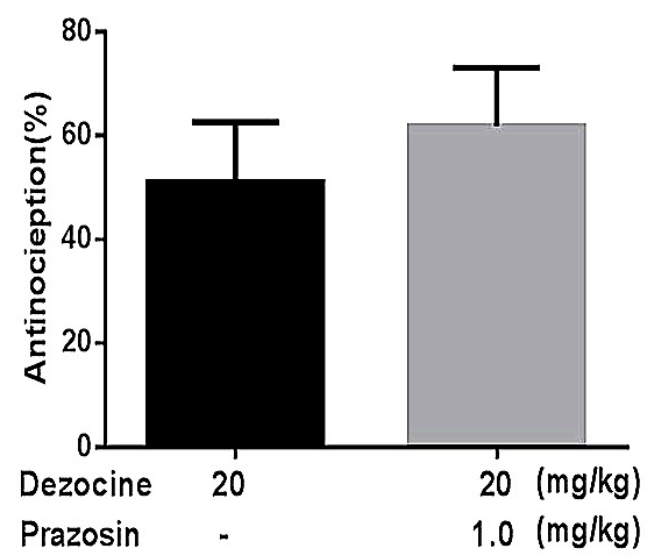

(b)

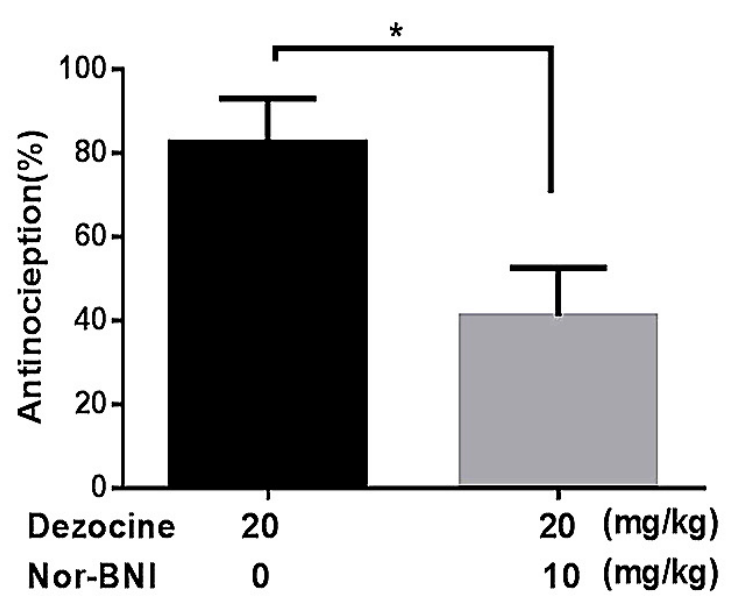

(d)

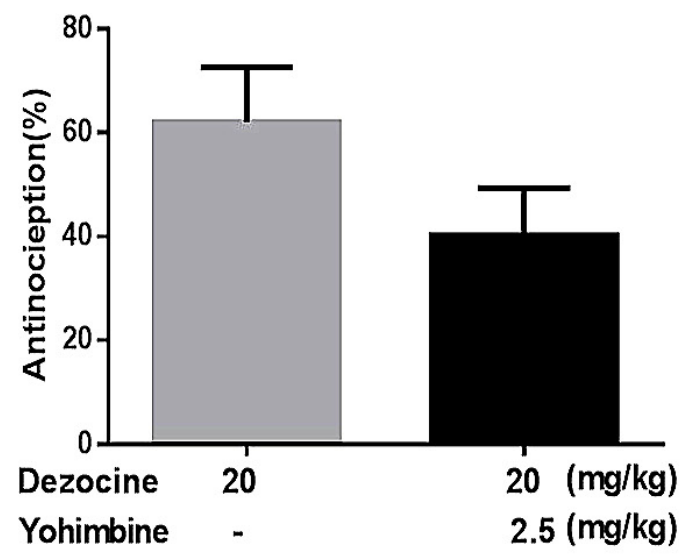

Figure 3.The analgesic effect of different doses of Dezocine and the effect of receptor antagonists on the analgesic effect of Dezocine

\subsection{Kappa opioid receptor antagonist significantly inhibited the analgesic effect of Dezocine in inflammatory pain}

Dezocine has a strong affinity with opioid receptors, especially Kappa opioid receptor. Previous experiments in our lab have shown that Dezocine's pain relief is produced by activating Kappa opioid receptor in the abdominal constriction test. In order to study which receptor was involved in the analgesic effect of Dezocine in inflammatory pain, we used the Kappa opioid receptor antagonist Nor-BNI, the selective $\alpha_{1}$ - norepinephrine receptor antagonist Prazosin, the selective $\alpha_{2}$ norepinephrine receptor were Yohimbine pretreated with mice. It was shown in figure 3 that intraperitoneal injection of Nor-BNI $(10 \mathrm{mg} / \mathrm{kg})$ could significantly antagonize the analgesic effect of Dezocine $(20 \mathrm{mg} / \mathrm{kg})$, while Prazosin $(1 \mathrm{mg} / \mathrm{kg})$ and Yohimbine $(2.5 \mathrm{mg} / \mathrm{kg})$ could not. The results indicated that Dezocine-produced analgesic effects in formalin-induced inflammatory pain was via Kappa opioid receptor.

\section{Discussion}

In this study, we demonstrated that Dezocine-induced analgesic effects was through activation of opioid receptors and inhibition of $\alpha_{2}$-norepinephrine uptake. The study helps understand the mechanism of Dezocine analgesia on models of acute nociceptive pain. First, we used the abdominal constriction test to investigate the mechanism of action of Dezocine. Previous laboratory studies have shown that Dezocine has a full dose analgesic effect in writhing experiments, both Nor-BNI and $\beta$-FNA (a selective $\mathrm{Mu}$ opioid receptor agonist) could significantly attenuated the analgesic effect of Dezocine[15]. In this study, we pretreated mice with different doses of Prazosin, Yohimbine, NAD 299, Altanserin, RS 102221, Ondansteron. Results showed that $1.25 \mathrm{mg} / \mathrm{kg} \alpha_{2}$ - norepinephrine receptor antagonist yohimbine significantly inhibiting the analgesic effect of Dezocine on acute pain, and 5-HT receptor antagonists did not affect the analgesic effect of Dezocine. Then, we investigated the role of Dezocine in the formalin-induce inflammatory pain model. Dezocine significantly reduced the number of times mice licking their feet after 
being injected with formalin, indicating that Dezocine produced potent analgesia. We pretreated mice with Kappa opioid receptor antagonist Nor-BNI, selective $\alpha_{1}$ norepinephrine receptor antagonist Prazosin, and selective $\alpha_{2}-$ norepinephrine receptor antagonist Yohimbine, only Nor-BNI was able to significantly attenuate the analgesic effects of Dezocine, whereas Prazosin and Yohimbine could not, suggesting that the mechanism of Dezocine in inflammatory pain relief is mediated by the activation of Kappa opioid receptor. Wang et al., in 2018 found that both $\mathrm{Mu}$ opioid receptor activation and norepinephrine reuptake mechanisms are linked to the action of Dezocine in cancer pain and neuropathic pain relief. However, in the present study, norepinephrine receptor was not involved in the inflammatory pain. This difference could be due to different animal models used and different mechanism involved. In addition, the serotonin antagonists were not used in the present study, and it is not yet clear whether serotonin plays a role in the analgesic effect of Dezocine.

\section{Acknowledgments}

The authors would like to thank Yangzi River Pharmaceuticals Group (Taizhou, Jiangsu, China) for providing Dezocine.

\section{References}

1. Loeser, J. D., Melzack, R. (1999) Pain: an overview, Lancet (London, England) 353, 1607-1609.

2. Woolf, C. J. (2010) What is this thing called pain?, The Journal of clinical investigation 120, 3742-3744.

3. Vanegas, G., Ripamonti, C., Sbanotto, A., De Conno, F. (1998) Side effects of morphine administration in cancer patients, Cancer nursing 21, 289-297.

4. McClung, C. A. (2006) The molecular mechanisms of morphine addiction, Reviews in the neurosciences 17, 393-402.

5. Kiyatkin, E. A. (2019) Respiratory depression and brain hypoxia induced by opioid drugs: Morphine, oxycodone, heroin, and fentanyl, Neuropharmacology 151, 219-226.

6. Sanansilp, V., Areewatana, S., Tonsukchai, N. (1998) Droperidol and the side effects of epidural morphine after cesarean section, Anesthesia and analgesia 86, 532-537.

7. Xiong, Z., Yi, P., Song, J., Tan, M. (2020) Dezocine prevents sufentanil-induced cough during general anesthesia induction: a meta-analysis of randomised controlled trials, BMC anesthesiology 20, 154.

8. Zhou, X., Zhang, C., Wang, M., Yu, L., Yan, M. (2015) Dezocine for Preventing Postoperative Pain: A Meta-Analysis of Randomized Controlled Trials, PloS one 10, e0136091.

9. Malis, J. L., Rosenthale, M. E., Gluckman, M. I. J. J. o. P., Therapeutics, E. (1975) Animal pharmacology of Wy-16,225, a new analgesic agent, 194, 488-498.

10. O'Brien, J. J., Benfield, P. (1989) Dezocine. A preliminary review of its pharmacodynamic and pharmacokinetic properties, and therapeutic efficacy, Drugs 38, 226-248.

11. Liu, R., Huang, X. P., Yeliseev, A., Xi, J., Roth, B. L. (2014) Novel molecular targets of dezocine and their clinical implications, Anesthesiology 120, 714-723.

12. Gharagozlou, P., Hashemi, E., DeLorey, T. M., Clark, J. D., Lameh, J. (2006) Pharmacological profiles of opioid ligands at kappa opioid receptors, BMC pharmacology 6, 3 .

13. Gharagozlou, P., Demirci, H., David Clark, J., Lameh, J. (2003) Activity of opioid ligands in cells expressing cloned mu opioid receptors, BMC pharmacology 3,1 .

14. Gharagozlou, P., Demirci, H., Clark, J. D., Lameh, J. (2002) Activation profiles of opioid ligands in HEK cells expressing delta opioid receptors, BMC neuroscience 3, 19.

15. Wang, Y. H., Chai, J. R., Xu, X. J., Ye, R. F., Zan, G. Y., Liu, G. Y., Long, J. D., Ma, Y., Huang, X., Xiao, Z. C., Dong, H., Wang, Y. J. (2018) Pharmacological Characterization of Dezocine, a Potent Analgesic Acting as a $\kappa$ Partial Agonist and $\mu$ Partial Agonist, Scientific reports 8, 14087.

16. Wang, Y. X., Mao, X. F., Li, T. F., Gong, N., Zhang, M. Z. (2017) Dezocine exhibits antihypersensitivity activities in neuropathy through spinal $\mu$-opioid receptor activation and norepinephrine reuptake inhibition, Scientific reports 7, 43137.

17. Barrett, A., C., Smith, E., S., Picker, M., Pharmacology, J. J. E. J. o. (2002) Sex-related differences in mechanical nociception and antinociception produced by $\mathrm{m}$ - and k-opioid receptor agonists in rats.

18. Swieboda, P., Filip, R., Prystupa, A., Drozd, M. (2013) Assessment of pain: types, mechanism and treatment, Annals of agricultural and environmental medicine : AAEM Spec no. 1, 2-7. 